\title{
Free Triiodothyronine and Free Triiodothyronine to Free Thyroxine Ratio Predict All-Cause Mortality in Patients with Diabetic Foot Ulcers
}

\author{
Jing Hong $\mathbb{D}^{\prime}, *$, Wen-Yue Liu',*, Xiang Hu', Wen-Wen Chen², Fei-Fei Jiang', Ze-Ru Xu', Fei-Xia Shen', \\ Hong Zhu (D) \\ 'Department of Endocrinology, The First Affiliated Hospital of Wenzhou Medical University, Wenzhou, 325000, People's Republic of China; \\ ${ }^{2}$ Department of Geriatrics, The First Affiliated Hospital of Wenzhou Medical University, Wenzhou, 325000, People's Republic of China \\ *These authors contributed equally to this work \\ Correspondence: Hong Zhu, Department of Endocrinology, The First Affiliated Hospital of Wenzhou Medical University, Wenzhou, People's Republic \\ of China, Tel +86 577-55579622, Fax +86 577-55578522, Email zhuhong@wmu.edu.cn
}

Purpose: Free triiodothyronine (FT3) and FT3/free thyroxine (FT4) ratio have been associated with mortality in various diseases. However, no study to date has identified a link between FT3, FT3/FT4 ratio and all-cause mortality in patients with diabetic foot ulcers (DFUs). This study aimed to investigate this relationship.

Methods: This retrospective cohort study included 726 patients diagnosed with DFUs in a public hospital from January 2015 to October 2019. Patients were classified by the optimal cut-off values of the FT3 and FT3/FT4 ratio, respectively. The association of FT3 and FT3/FT4 ratio with all-cause mortality was evaluated in a multivariable cox regression model. Directed acyclic graphs were used to assess the minimally sufficient sets of confounding variables.

Results: Log rank tests indicated that patients with low FT3 and FT3/FT4 ratio had lower overall survival rates (all p $<0.001)$. The adjusted HRs for all-cause mortality were 0.48 (95\% CI: $0.32-0.73, \mathrm{P}=0.001)$ when comparing high versus low FT3 and 0.47 (95\% CI: $0.32-0.70, \mathrm{P}<0.001)$ when comparing high versus low FT3/FT4 ratio. Subgroup analyses showed that these associations existed only in elderly patients ( $\geq 65$ years) and women, after adjustment. In men, only high FT3/FT4 ratio was associated with low all-cause mortality, after adjustment.

Conclusion: Routine assessment of FT3 and FT3/FT4 ratio may be a simple and effective way to identify high-risk patients with DFUs, especially in elderly patients and women.

Keywords: FT3, FT3/FT4 ratio, mortality, diabetic foot ulcer

\section{Introduction}

Diabetic foot ulcers (DFUs), a common and disastrous complication of diabetes mellitus, ${ }^{1}$ are the cause of a high rate of amputation and health-care costs and are generally associated with an excess risk of all-cause mortality., Patients with DFUs face a 5-year mortality as high as $30.5 \%$, which is comparable to cancer. ${ }^{4}$ The occurrence and prognosis of DFUs are related to many factors, such as genetic factors, ${ }^{5}$ smoking ${ }^{6}$ and therapeutic interventions, for instance, autologous platelet-rich gel. ${ }^{7}$ Several risk factors were found to contribute to the mortality of DFUs, including age, male, chronic renal insufficiency, dialysis, lower hemoglobinA1c (HbA1c), peripheral artery disease and the severity of DFUs. ${ }^{3,8,9}$ However, the risk factors were inadequate and inconsistent in different population. ${ }^{89}$ Identifying other commonly available clinical factors that predict mortality in patients with DFUs is important to improve outcomes and prolong life.

Attention has been recently devoted to thyroid hormones as factors in predicting mortality in various diseases, especially acute or systemic diseases. Lower triiodothyronine (T3) was found to be closely associated with mortality in patients with chronic renal failure (CRF), ${ }^{10}$ acute myocardial infarction ${ }^{11}$ and surgical sepsis. ${ }^{12}$ The FT3/FT4 ratio was 
a marker of thyroid hormone conversion, reflecting deiodinase activity. ${ }^{13}$ Lower FT3/FT4 ratio was found to be associated with mortality in patients with coronary artery disease, ${ }^{14}$ patients undergoing PCI, ${ }^{15}$ patients with dilated cardiomyopathy and ${ }^{16}$ colorectal cancer ${ }^{17}$ and hospitalized geriatric patients. ${ }^{18}$ DFUs as a severe diabetic complication also accompany significant systemic dysfunction. Poor glycemic control, diabetic neuropathy and peripheral artery disease neuropathy are known as causative factors of DFUs. ${ }^{3}$ Studies ${ }^{19-24}$ have found that FT3 was associated with glycemic control, diabetes, diabetic retinopathy, diabetic nephropathy and diabetic microangiopathy. High FT3/FT4 ratio was associated with diabetes. ${ }^{20}$ Therefore, there might be a potential relationship between FT3, FT3/FT4 ratio and the prognosis of DFUs.

Up to now, no study has identified a relationship between FT3, FT3/FT4 ratio and all-cause mortality in patients with DFUs. Therefore, in this study, we aimed to explore the role of FT3 and FT3/FT4 ratio in predicting the risk of all-cause mortality in patients with DFUs.

\section{Materials and Methods Study Population}

This retrospective cohort study enrolled 907 participants who were diagnosed with type 2 diabetes mellitus and DFUs in the First Affiliated Hospital of Wenzhou Medical University from January 2015 to October 2019. The exclusion criteria included those with previous thyroid disease and persons under medication interfering with thyroid function (thyroid hormone preparations, amiodarone, methimazole, propylthiouracil, antipsychotics and lithium). Finally, a total of 726 patients with DFUs were included in this study.

The study protocol was approved by the ethics committee of the First Affiliated Hospital of Wenzhou Medical University (approval number: 2018-105). The data of patients accessed were anonymized and maintained with confidentiality. Due to the retrospective nature of the study, not compromising the privacy of patients, the requirement for informed consent was waived. This study was performed in accordance with the Declaration of Helsinki as revised in 2013.

\section{Data Collection and Grouping}

The baseline information collected including demographic characteristics, diabetes duration, hypertension, lifestyle habits including smoking, alcohol use and laboratory parameters including free thyroxine (FT4), free triiodothyronine (FT3), thyroid-stimulating hormone (TSH), albumin (ALB), hemoglobin ( $\mathrm{Hb}$ ), HbA1c, total cholesterol (TC), triglyceride (TG), high-density lipoprotein cholesterol (HDL-C), low-density lipoprotein cholesterol (LDL-C) and creatinine were retrospectively extracted from medical records. The primary end point for this study was all-cause mortality. Survival status was acquired through medical records or telephone follow-up. The Chronic Kidney Disease Epidemiology Collaboration equation was used to calculate the estimated glomerular filtration rate (eGFR). ${ }^{25}$ Smoking was categorized as currently smoking or non-smoking (including ex-smokers). Alcohol use was categorized as currently drinking or no alcohol consumption. Individuals $\geq 65$ years old were defined as elderly, according to the United Nations definition. The degree of severity of DFUs was subdivided according to the Wagner classification ${ }^{26}$ (grade score $0-5$ ) and categorized as no severe DFUs (Wagner grade score $<3$ ) and severe DFUs (Wagner grade score $\geq 3$ ). Patients were categorized as low FT3, high FT3 groups and low FT3/FT4 ratio, high FT3/FT4 ratio groups based on the optimal cut-off values of the FT3 and FT3/FT4 ratio suggested by the ROC curves.

\section{Statistical Analysis}

The data are presented as $\mathrm{n}(\%)$, mean \pm standard deviation, median and interquartile range (skewed variables). Continuous variables were compared using two-sided Student's $t$-test or Mann-Whitney $U$-test. Categorical variables were compared using Chi-squared test. The cut-off values of FT3 and FT3/FT4 ratio for the all-cause mortality were evaluated using the receiver operating characteristic (ROC) curves. The cut-off value with the highest sum of sensitivity and specificity was considered as the optimal cut-off value. Kaplan-Meier survival curves and log rank tests were used to compare the differences in overall survival (OS). The relationship between FT3, FT3/FT4 ratio and 
all-cause mortality was analyzed by cox proportional hazards regression. HRs with corresponding 95\% CIs were estimated. An online DAGitty software (http://www.dagitty.net) was used to construct directed acyclic graphs (DAGs) (Supplemental Figures 1 and 2) and assess the minimally sufficient sets of confounding variables, ${ }^{27}$ which included age, sex, severe DFUs, smoking, alcohol use, diabetes duration, eGFR and HbA1c for FT3. The variables for FT3/FT4 ratio were age, sex, smoking, alcohol use, eGFR and HbA1c. P values of 0.05 were considered statistically significant for all tests. The statistical analyses were performed using SPSS (IBM, IL, USA) version 22.

\section{Results}

\section{Clinical Characteristics of the Study Population}

The optimal cut-off values of FT3 and FT3/FT4 ratio for the all-cause mortality suggested by ROC curves were 3.72 (pmol/L) and 0.3068, respectively. Area under ROC curve (AUC) for FT3 was 0.608, 95\% CI: 0.549-0.668. AUC for FT3/FT4 ratio was $0.593,95 \%$ CI: $0.534-0.653$ (Figure 1). The clinical characteristics of the study population subdivided according to the optimal cut-off values of FT3 and FT3/FT4 ratio are shown in Tables 1 and 2. Patients with low FT3 had longer diabetes duration, higher prevalence of severe DFUs, HbA1c and lower eGFR, ALB, Hb, TC, HDL-C, and LDL$\mathrm{C}$ than those with high FT3 (all P $<0.05$ ). Patients with low FT3/FT4 ratio had higher HbA1c, prevalence of Wagner grade score $\geq 3$ and lower ALB, Hb, TC, TG, HDL-C, LDL-C, and prevalence of alcohol use than those with high FT3/ FT4 ratio (all $\mathrm{P}<0.05$ ).

\section{Kaplan-Meier Curves for OS}

Log rank tests of the Kaplan-Meier curves indicated that patients with low FT3 and FT3/FT4 ratio had lower OS rates compared to patients with high FT3 and FT3/FT4 ratio (Figure 2). The cumulative overall survival rates at 1, 3, and 5 years are shown in Table 3 .

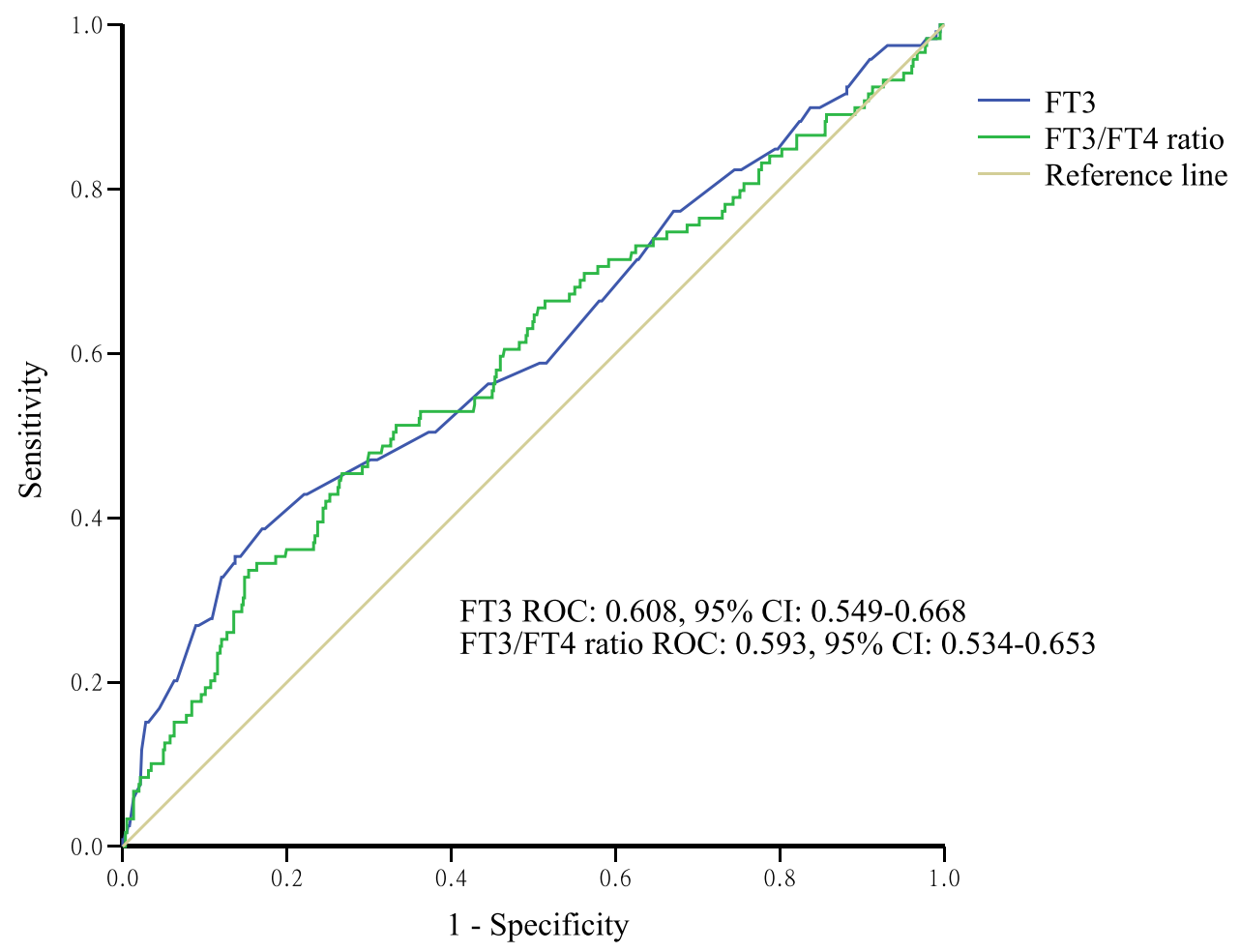

Figure I ROC curves of FT3 and FT3/FT4 ratio for predicting the all-cause mortality. The optimal cut-off values of FT3 and FT3/FT4 ratio were 3.72 (pmol/L) and 0.3068, respectively. AUC for FT3 was $0.608,95 \% \mathrm{Cl}$ : 0.549-0.668. AUC for FT3/FT4 ratio was 0.593, 95\% Cl: $0.534-0.653$.

Abbreviations: FT3, free triiodothyronine; FT4, free thyroxine; ROC, receiver operating characteristic; AUC, area under ROC curve. 

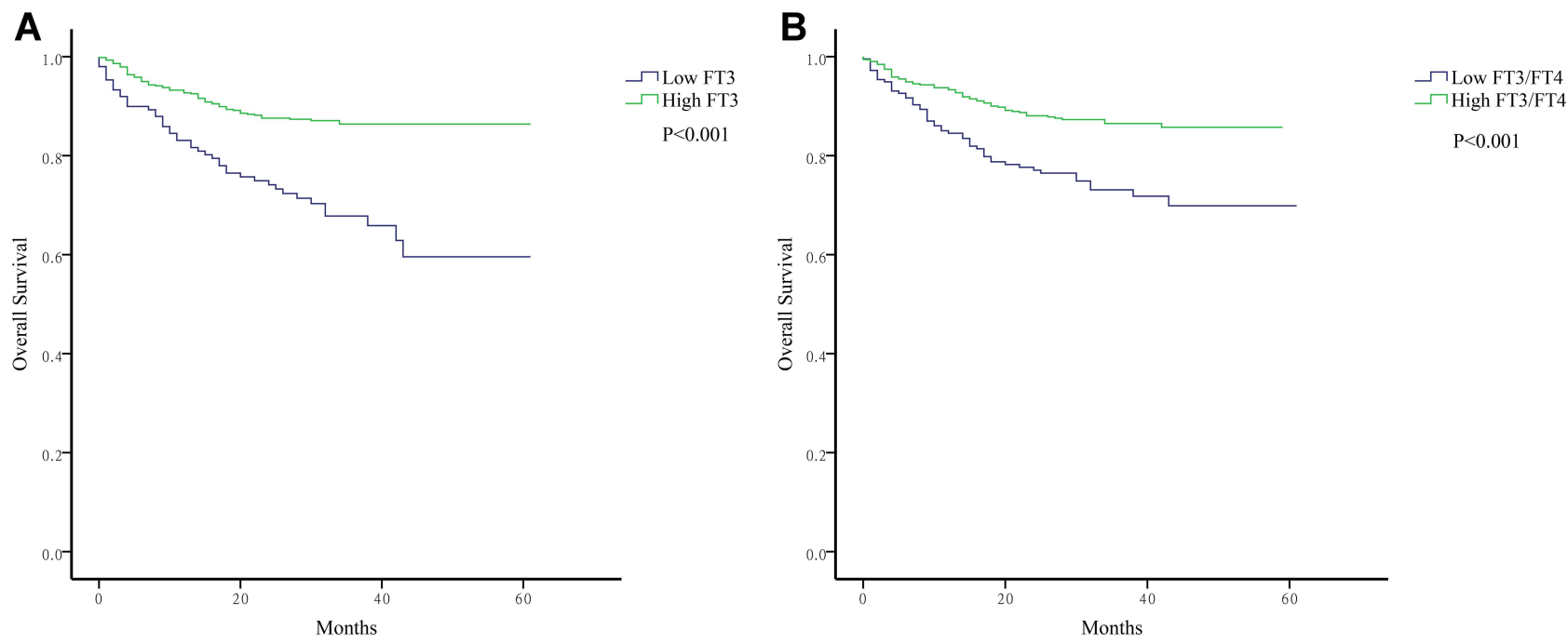

Figure 2 Kaplan-Meier curve of overall survival. (A) Low FT3 (<3.72 pmol/L) and High FT3 ( $\geq 3.72$ pmol/L). (B) Low FT3/FT4 ratio (<0.3068) and High FT3/FT4 ratio $(\geq 0.3068)$

\section{Unadjusted and Multivariate Cox Regression Analyses for All-Cause Mortality}

The unadjusted and multivariable-adjusted cox regression analyses were performed to evaluate the relationship between FT3, FT3/FT4 ratio and all-cause mortality (Table 4). The adjusted HRs for all-cause mortality were 0.48 (95\% CI: 0.32 $0.73, \mathrm{P}=0.001)$ when comparing high versus low FT3 and $0.47(95 \% \mathrm{CI}: 0.32-0.70, \mathrm{P}<0.001)$ when comparing high versus low FT3/FT4 ratio. In subgroup analyses stratified by gender, similar results were found in woman: high FT3

Table I Baseline Characteristics of Participants with Low and High FT3

\begin{tabular}{|c|c|c|c|}
\hline Characteristic & Low FT3 $(<3.72 \mathrm{pmol} / \mathrm{L})(\mathrm{N}=149)$ & High FT3 $(\geq 3.72 \mathrm{pmol} / \mathrm{L})(\mathrm{N}=577)$ & P-value \\
\hline Male (\%) & $89(59.7)$ & $343(59.4)$ & 0.949 \\
\hline Age (years) & $68(6 I-76)$ & $69(60-76)$ & 0.705 \\
\hline Age $\geq 65$ (\%) & $95(63.8)$ & $361(62.6)$ & 0.788 \\
\hline Smoking (\%) & $42(28.2)$ & $170(29.5)$ & 0.760 \\
\hline Alcohol use (\%) & $39(26.2)$ & $144(25.0)$ & 0.760 \\
\hline Diabetes duration (years) & $10(10-20)$ & $10(5-18)$ & 0.002 \\
\hline Diabetic foot ulcer duration (days) & $30(10-60)$ & $30(10-60)$ & 0.225 \\
\hline Severe DFUs (\%) & $100(67.1)$ & $288(49.9)$ & $<0.001$ \\
\hline $\mathrm{SBP}(\mathrm{mmHg})$ & $138(123-158)$ & $143(128-159)$ & 0.066 \\
\hline $\mathrm{DBP}(\mathrm{mmHg})$ & $73(64-83)$ & $75(68-83)$ & 0.122 \\
\hline TSH (mlU/L) & $1.55(0.93-2.7 \mathrm{I})$ & $1.49(0.94-2.22)$ & 0.223 \\
\hline FT3 (pmol/L) & $3.30(3.10-3.53)$ & $4.40(4.10-4.70)$ & $<0.001$ \\
\hline FT4 (pmol/L) & $12.05(10.13-13.73)$ & $11.83(10.63-13.84)$ & 0.686 \\
\hline FT3/FT4 ratio & $0.27(0.23-0.33)$ & $0.37(0.32-0.43)$ & $<0.001$ \\
\hline eGFR (EPI) $\left(\mathrm{mL} / \mathrm{min} / 1.73 \mathrm{~m}^{2}\right)$ & $73.7(45.0-92.8)$ & $83.6(61.5-96.1)$ & 0.002 \\
\hline ALB $(g / L)$ & $29.3 \pm 5.7$ & $34.6 \pm 4.8$ & $<0.001$ \\
\hline $\mathrm{Hb}(\mathrm{g} / \mathrm{L})$ & $102.5 \pm 17.1$ & $118.0 \pm 17.7$ & $<0.001$ \\
\hline HbAlc (\%) & $8.9(7.6-11.5)$ & $8.6(7.4-10.5)$ & 0.037 \\
\hline $\mathrm{TC}(\mathrm{mmol} / \mathrm{L})$ & $3.70(3.08-4.52)$ & $4.07(3.34-5.10)$ & 0.003 \\
\hline TG (mmol/L) & $1.22(0.84-1.64)$ & $1.28(0.94-1.82)$ & 0.157 \\
\hline HDL-C (mmol/L) & $0.76(0.57-0.99)$ & $0.92(0.75-1.1 \mathrm{I})$ & $<0.001$ \\
\hline LDL-C (mmol/L) & $2.13(1.65-2.69)$ & $2.37(1.72-3.08)$ & 0.023 \\
\hline
\end{tabular}

Abbreviations: DFUs, diabetic foot ulcers; SBP, systolic blood pressure; DBP, diastolic blood pressure; TSH, thyroid-stimulating hormone; FT3, free triiodothyronine; T4, thyroxine; FT4, free thyroxine; eGFR, estimated glomerular filtration rate; ALB, albumin; Hb, hemoglobin; HbAlc, hemoglobin Alc; TC, total cholesterol; TG, triglyceride; HDL, high-density lipoprotein; LDL, low-density lipoprotein. 
Table 2 Baseline Characteristics of Participants with Low and High FT3/FT4 Ratio

\begin{tabular}{|c|c|c|c|}
\hline Characteristic & Low FT3/FT4 ratio $(<0.3068)(\mathrm{N}=216)$ & High FT3/FT4 ratio $(\geq 0.3068)(N=510)$ & P-value \\
\hline Male (\%) & $117(54.2)$ & $315(61.8)$ & 0.057 \\
\hline Age (years) & $70(60-77)$ & $68(60-75)$ & 0.110 \\
\hline Age $\geq 65$ (\%) & $145(67.1)$ & $311(6 I .0)$ & 0.177 \\
\hline Smoking (\%) & $61(28.2)$ & $15 \mid(29.6)$ & 0.711 \\
\hline Alcohol use (\%) & $43(19.9)$ & $140(27.5)$ & 0.032 \\
\hline Diabetes duration (years) & $10(6-20)$ & $10(6-18)$ & 0.405 \\
\hline Diabetic foot ulcer duration (days) & $30(10-60)$ & $30(10-60)$ & 0.347 \\
\hline Severe DFUs (\%) & $149(69.0)$ & $239(46.9)$ & $<0.001$ \\
\hline SBP $(\mathrm{mmHg})$ & $14 \mid(\mid 25-158)$ & $144(128-159)$ & 0.231 \\
\hline $\mathrm{DBP}(\mathrm{mmHg})$ & $74(65-83)$ & $75(68-83)$ & 0.400 \\
\hline TSH (mIU/L) & $1.31(0.75-2.14)$ & $1.57(1.05-2.40)$ & $<0.001$ \\
\hline FT3 (pmol/L) & $3.80(3.23-4.10)$ & $4.40(4.00-4.80)$ & $<0.001$ \\
\hline FT4 (pmol/L) & $14.39(13.13-16.19)$ & $11.20(10.03-12.33)$ & $<0.001$ \\
\hline FT3/FT4 ratio & $0.26(0.23-0.29)$ & $0.38(0.35-0.44)$ & $<0.001$ \\
\hline eGFR (EPI) $\left(\mathrm{mL} / \mathrm{min} / 1.73 \mathrm{~m}^{2}\right)$ & $80.5(56.3-94.9)$ & $82.6(58.4-96.0)$ & 0.333 \\
\hline ALB (g/L) & $30.4 \pm 5.6$ & $34.8 \pm 4.8$ & $<0.001$ \\
\hline $\mathrm{Hb}(\mathrm{g} / \mathrm{L})$ & $105.6 \pm 17.8$ & $118.7 \pm 17.6$ & $<0.001$ \\
\hline HbAlc (\%) & $8.9(7.6-11.5)$ & $8.6(7.3-10.4)$ & 0.005 \\
\hline $\mathrm{TC}(\mathrm{mmol} / \mathrm{L})$ & $3.70(3.04-4.59)$ & $4.13(3.42-5.10)$ & $<0.001$ \\
\hline TG (mmol/L) & $1.20(0.84-1.62)$ & $1.29(0.95-1.86)$ & 0.013 \\
\hline HDL-C (mmol/L) & $0.80(0.63-1.02)$ & $0.93(0.76-I . I I)$ & $<0.001$ \\
\hline LDL-C (mmol/L) & $2.11(1.67-2.78)$ & 2.37 (1.74-3.07) & 0.021 \\
\hline
\end{tabular}

Abbreviations: DFUs, diabetic foot ulcers; SBP, systolic blood pressure; DBP, diastolic blood pressure; TSH, thyroid-stimulating hormone; FT3, free triiodothyronine; T4, thyroxine; FT4, free thyroxine; eGFR, estimated glomerular filtration rate; ALB, albumin; Hb, hemoglobin; HbAlc, hemoglobin Alc; TC, total cholesterol; TG, triglyceride; HDL, high-density lipoprotein; LDL, low-density lipoprotein.

Table 3 Cumulative Overall Survival of Participants Categorized by FT3 and FT3/FT4 Ratio

\begin{tabular}{|l|l|l|l|}
\hline & I-Year OS & 3-Year OS & 5-Year OS \\
\hline FT3 & & & \\
Low (<3.72 pmol/L) & $83.1 \%$ & $67.8 \%$ & $59.6 \%$ \\
High ( $\geq 3.72 \mathrm{pmol} / \mathrm{L})$ & $92.7 \%$ & $86.4 \%$ & $86.4 \%$ \\
FT3/FT4 ratio & & & \\
Low $(<0.3068)$ & $84.5 \%$ & $73.1 \%$ & $69.9 \%$ \\
High $(\geq 0.3068)$ & $93.3 \%$ & $86.5 \%$ & $85.7 \%$ \\
\hline
\end{tabular}

Abbreviations: FT3, free triiodothyronine; FT4, free thyroxine; OS, overall survival.

(adjusted HRs 0.38, 95\% CI: 0.21-0.72, P = 0.003) and FT3/FT4 ratio (adjusted HRs 0.39, 95\% CI: 0.21-0.71, P = 0.002) were inversely associated with all-cause mortality. Unadjusted cox regression analyses revealed that high FT3 and FT3/FT4 ratio were associated with low all-cause mortality in men; however, only the association between high FT3/FT4 ratio (adjusted HRs $0.54,95 \% \mathrm{CI}: 0.32-0.91, \mathrm{P}=0.021$ ) and low all-cause mortality remained significant after adjusting by confounding variables. In subgroup analyses stratified by age, high FT3 (adjusted HRs 0.44, 95\% CI: 0.28-0.69, P < 0.001 ) and FT3/FT4 ratio (adjusted HRs 0.40, 95\% CI: 0.26-0.62, P $<0.001$ ) were associated with low all-cause mortality only in elderly patients ( $\geq 65$ years), after adjustment.

Cox regression analyses were also used to evaluate the relationship between TSH, FT4 and all-cause mortality. No significant association was found between TSH, FT4 and all-cause mortality $(\mathrm{P}>0.05)$. 
Table 4 Unadjusted and Multivariate Cox Regression Analyses for All-Cause Mortality

\begin{tabular}{|c|c|c|c|c|}
\hline & Unadjusted HR (95\% Cl) & P-value & Adjusted HR (95\% Cl) & P-value \\
\hline \multicolumn{5}{|l|}{ Total } \\
\hline \multicolumn{5}{|l|}{ FT3 } \\
\hline Low (<3.72 pmol/L) & Ref & - & Ref & - \\
\hline High ( $\geq 3.72 \mathrm{pmol} / \mathrm{L})$ & $0.37(0.25-0.53)$ & $<0.001$ & $0.48(0.32-0.73)^{\mathrm{a}}$ & 0.001 \\
\hline \multicolumn{5}{|l|}{ FT3/FT4 ratio } \\
\hline Low $(<0.3068)$ & Ref & - & Ref & - \\
\hline High $(\geq 0.3068)$ & $0.46(0.32-0.66)$ & $<0.001$ & $0.47(0.32-0.70)^{b}$ & $<0.001$ \\
\hline \multicolumn{5}{|l|}{ Male } \\
\hline \multicolumn{5}{|l|}{ FT3 } \\
\hline Low (<3.72 pmol/L) & Ref & - & Ref & - \\
\hline High ( $\geq 3.72$ pmol/L) & $0.46(0.28-0.75)$ & 0.002 & $0.61(0.35-1.07)^{c}$ & 0.082 \\
\hline \multicolumn{5}{|l|}{ FT3/FT4 ratio } \\
\hline Low $(<0.3068)$ & Ref & - & Ref & - \\
\hline High $(\geq 0.3068)$ & $0.50(0.3 \mathrm{I}-0.8 \mathrm{I})$ & 0.005 & $0.54(0.32-0.91)^{d}$ & 0.021 \\
\hline \multicolumn{5}{|l|}{ Female } \\
\hline \multicolumn{5}{|l|}{ FT3 } \\
\hline Low (<3.72 pmol/L) & Ref & - & Ref & - \\
\hline High ( $(23.72 \mathrm{pmol} / \mathrm{L})$ & $0.27(0.15-0.47)$ & $<0.001$ & $0.38(0.21-0.72)^{c}$ & 0.003 \\
\hline \multicolumn{5}{|l|}{ FT3/FT4 ratio } \\
\hline Low $(<0.3068)$ & Ref & - & Ref & _ \\
\hline High $(\geq 0.3068)$ & $0.40(0.23-0.70)$ & 0.001 & $0.39(0.21-0.7 I)^{d}$ & 0.002 \\
\hline \multicolumn{5}{|l|}{ Age $<65$ years } \\
\hline \multicolumn{5}{|l|}{ FT3 } \\
\hline Low (<3.72 pmol/L) & Ref & - & Ref & - \\
\hline High ( $\geq 3.72 \mathrm{pmol} / \mathrm{L})$ & $0.33(0.13-0.82)$ & 0.017 & $0.83(0.28-2.43)^{\mathrm{e}}$ & 0.730 \\
\hline \multicolumn{5}{|l|}{ FT3/FT4 ratio } \\
\hline Low $(<0.3068)$ & Ref & - & Ref & _ \\
\hline High ( $\geq 0.3068)$ & $1.03(0.37-2.85)$ & 0.960 & $1.36(0.40-4.59)^{f}$ & 0.622 \\
\hline \multicolumn{5}{|l|}{ Age $\geq 65$ years } \\
\hline \multicolumn{5}{|l|}{ FT3 } \\
\hline Low (<3.72 pmol/L) & Ref & - & Ref & - \\
\hline High ( $\geq 3.72 \mathrm{pmol} / \mathrm{L})$ & $0.36(0.24-0.55)$ & $<0.001$ & $0.44(0.28-0.69)^{\mathrm{e}}$ & $<0.001$ \\
\hline \multicolumn{5}{|l|}{ FT3/FT4 ratio } \\
\hline Low $(<0.3068)$ & Ref & - & Ref & - \\
\hline High $(\geq 0.3068)$ & $0.4 \mathrm{I}(0.28-0.6 \mathrm{I})$ & $<0.001$ & $0.40(0.26-0.62)^{f}$ & $<0.001$ \\
\hline
\end{tabular}

Notes: ${ }^{a}$ The multivariable cox regression was adjusted for risk factors including age, sex, severe DFUs, smoking, alcohol use, diabetes duration, eGFR and HbAlc. ${ }^{\mathrm{b}}$ The multivariable cox regression was adjusted for risk factors including age, sex, smoking, alcohol use, eGFR and HbAlc. ${ }^{\mathrm{C}}$ The multivariable cox regression was adjusted for risk factors including severe DFUs, smoking, alcohol use, diabetes duration, eGFR and HbAlc. ${ }^{\mathrm{d}}$ The multivariable cox regression was adjusted for risk factors including age, smoking, alcohol use, eGFR and HbAlc. ${ }^{~} T$ The multivariable cox regression was adjusted for risk factors including sex, severe DFUs, smoking, alcohol use, diabetes duration, eGFR and $\mathrm{HbAlc}$. ${ }^{\mathrm{T}}$ The multivariable cox regression was adjusted for risk factors including age, sex, smoking, alcohol use, eGFR and HbAlc.

Abbreviations: DFUs, diabetic foot ulcers; FT3, free triiodothyronine; FT4, free thyroxine; eGFR, estimated glomerular filtration rate; HbAIc, hemoglobin Alc; Ref, reference.

\section{Discussion}

To the best of our knowledge, this is the first study to reveal the relationship between FT3, FT3/FT4 ratio and all-cause mortality in patients with DFUs. In this study, log rank tests of the Kaplan-Meier curves indicated that patients with low FT3 and FT3/FT4 ratio had lower OS rates. This study demonstrated an independent association between low FT3, FT3/ FT4 ratio and high all-cause mortality in patients with DFUs by multivariable cox regression analyses.

In previous studies, T3, thyroxine (T4), FT3/FT4 ratio and TSH had different predictive effects on mortality. Low FT3, FT3/FT4 ratio, high FT4 but not TSH were found to be associated with high mortality in older adults. ${ }^{18,28}$ Low FT3/FT4 ratio was found to be associated with high mortality in patients who underwent PCI $^{15}$ and with dilated cardiomyopathy and ${ }^{16}$ colorectal cancer, ${ }^{17}$ but these studies have not evaluated the relationship between FT3, FT4, 
TSH and mortality. Low FT3, high FT4 but not TSH were found to be associated with high mortality in patients who underwent coronary angiography. ${ }^{29}$ Distinct clinical and anthropometric characteristics of participants might account for the conflicting results of these studies.

The pathophysiological mechanism underlying the association between low FT3, FT3/FT4 ratio and high all-cause mortality in patients with DFUs remains unclear. Most of the circulating T3 is produced from peripheral thyroxine deiodination. Thus, T3 exerts a potent biological effect on targeted organs and tissues. ${ }^{30}$ Conversion of FT4 to FT3 takes place in the liver, kidneys, skeletal muscles and thyroid. ${ }^{31}$ Reduced renal blood flow, poor nutritional status, peripheral metabolic changes and increased pro-inflammatory cytokines may result in low T3 levels. ${ }^{16}$ FT3/FT4 ratio reflects the 5'deiodinase activity, and impaired peripheral deiodinase activity may be an early physiopathologic reaction to various diseases. ${ }^{32}$ FT3/FT4 ratio was also suggested as an indicator of unfavorable metabolic profiles. ${ }^{33}$ Some of our participants may be accompanied by euthyroid sick syndrome, which is characterized by low T3 in people with normal thyroid function. Euthyroid sick syndrome is common in critically ill patients or people in poor nutritional status. The down-regulation of FT3 levels was thought to be an adaptive mechanism to acute or serious diseases. ${ }^{32,34}$ On the basis of discussions above, it can be concluded that patients with low FT3 and FT3/FT4 ratio might represent a subgroup of people with serious diseases related to high all-cause mortality.

In this study, patients with low FT3 and FT3/FT4 ratio were in an unfavorable condition: poor nutritional status (lower $\mathrm{Hb}$ and $\mathrm{ALB}$ ), heavier burden of disease (higher prevalence of severe DFUs), and worse glycemic control (higher HbA1c). A previous study ${ }^{18}$ also found that FT3 and FT3/FT4 ratio positively correlated with Hb and ALB in hospitalized older patients. Partly consistent with previous reports, ${ }^{33,35}$ in which FT3 and FT3/FT4 ratio were positively related to TG and glucose and negatively with HDL-C, in the present study, patients with low FT3 had lower TC, HDL$\mathrm{C}$, and LDL-C, and patients with low FT3/FT4 ratio had lower TC, TG, HDL-C, and LDL-C. Low T3 is the most frequent alteration of the thyroid hormone observed in chronic kidney disease, due to reduced deiodinase activity, inorganic iodide accumulation, and toxic uremic solutes, altering the central control of the pituitary gland. ${ }^{36}$ In this study, participants with low FT3 had significantly lower eGFR. Similarly, lower eGFR was observed in participants with low FT3/FT4 ratio, but the difference was small and did not reach statistical significance.

In subgroup analyses, the association between high FT3 and low all-cause mortality became no longer statistically significant after adjustment for confounders in men. A prospective cohort study of the outpatients $\geq 75$ years old in Italy ${ }^{37}$ has reported a similar finding that a significant association between FT3 and all-cause mortality was only found in women, not in men. They also found that low TSH and high FT4 in men were associated with high mortality. However, no association was found between FT4 and all-cause mortality in this study. The associations between high FT3/FT4 ratio and low all-cause mortality were found in both women and men. FT3/FT4 ratio might be a more effective predictor of all-cause mortality than a single thyroid hormone in men. The mechanism behind the gender difference is unclear. One of the probable explanations is that thyroid diseases, such as autoimmune disease and subclinical thyroid dysfunctions, are more prevalent in women than in men, ${ }^{38}$ which might lead to an enhanced mortality. It is hypothesized that sexual chromosomes and/or gonadal hormones might deeply affect thyroid physiology and pathophysiology. ${ }^{38}$ In addition, although the odds of having severe DFUs such as osteomyelitis were irrespective of gender, ${ }^{39}$ gender was found to be a predictor of mortality of DFUs. ${ }^{9}$

In subgroup analyses stratified by age, high FT3 and FT3/FT4 ratio were only associated with low all-cause mortality in elderly patients ( $\geq 65$ years). Previous study ${ }^{40}$ shows that thyroid dysfunction arises with age, which is associated with increased mortality. The researchers consider that the consequences of thyroid dysfunction during middle age and elderly were inconsistent and cannot be extrapolated to each other. ${ }^{40}$ FT3 declines with age, which is likely due to the decrease in peripheral T4 to T3 conversion. This might be an important contributor to the decrease in basal metabolic rate with aging. ${ }^{41}$ Euthyroid sick syndrome is also common in the elderly. ${ }^{42}$ The above conditions might contribute to the discrepancies in results between patients $\geq 65$ years old and $<65$ years old.

This study has two strengths. First, the sample size of this study was relatively large. Second, in order to avoid bias, DAGs were used to guide the construction of multivariable cox regression to ensure that only plausible confounders were adjusted. 
This study also has a limitation. The participants in this study were from single center. Therefore, the results may not be applicable to general patients with DFUs. Further multi-center research will be needed to complement the limitation.

\section{Conclusion}

This study identified FT3 and FT3/FT4 ratio as new prognostic factors in patients with DFUs. Routine assessment of FT3 and FT3/FT4 ratio is a simple and effective way to identify high-risk patients with DFUs, especially in elderly patients and women, and early intensive interventions may help to improve the prognosis of those patients.

\section{Abbreviations}

DFUs, diabetic foot ulcers; T3, triiodothyronine; FT3, free triiodothyronine; T4, thyroxine; FT4, free thyroxine; TSH, thyroid-stimulating hormone; SBP, systolic blood pressure; DBP, diastolic blood pressure; HDL, high-density lipoprotein; LDL, low-density lipoprotein; TC, total cholesterol; TG, triglyceride; HbA1c, hemoglobin A1c; ALB, albumin; Hb, hemoglobin; eGFR, estimated glomerular filtration rate; OS, overall survival; DAG, directed acyclic graph; ROC, receiver operating characteristic; AUC, area under ROC curve; Ref, Reference.

\section{Data Sharing Statement}

The datasets used and analysed during the current study are available from the corresponding author on reasonable request.

\section{Ethics Approval and Consent to Participate}

The study protocol was approved by the ethics committee of the First Affiliated Hospital of Wenzhou Medical University (approval number: 2018-105). The data of patients accessed were anonymized and maintained with confidentiality. Due to the retrospective nature of the study, not compromising the privacy of patients, the requirement for informed consent was waived. This study was performed in accordance with the Declaration of Helsinki as revised in 2013.

\section{Acknowledgments}

The authors like to acknowledge all the patients who participated in the study.

\section{Funding}

This study was supported by the Natural Science Foundation of China (81900737), the Basic Scientific Research Program of Wenzhou Medical University, China (KYYW202015).

\section{Disclosure}

The authors have no conflicts of interest to disclose.

\section{References}

1. Singh N, Armstrong DG, Lipsky BA. Preventing foot ulcers in patients with diabetes. JAMA. 2005;293(2):217-228. doi:10.1001/jama.293.2.217

2. Brownrigg JR, Davey J, Holt PJ, et al. The association of ulceration of the foot with cardiovascular and all-cause mortality in patients with diabetes: a meta-analysis. Diabetologia. 2012;55(11):2906-2912. doi:10.1007/s00125-012-2673-3

3. Jalilian M, Ahmadi Sarbarzeh P, Oubari S. Factors related to severity of diabetic foot ulcer: a systematic review. Diabetes Metabol Syndr Obes. 2020;13:1835-1842. doi:10.2147/dmso.S256243

4. Armstrong DG, Swerdlow MA, Armstrong AA, Conte MS, Padula WV, Bus SA. Five year mortality and direct costs of care for people with diabetic foot complications are comparable to cancer. J Foot Ankle Res. 2020;13(1):16. doi:10.1186/s13047-020-00383-2

5. Zhao J, Zhang LX, Wang YT, Li Y, Chen HL. Genetic polymorphisms and the risk of diabetic foot: a systematic review and meta-analyses. Int J Low Extrem Wounds. 2020;1534734620977599. doi:10.1177/1534734620977599

6. Fu XL, Ding H, Miao WW, Chen HL. Association between cigarette smoking and diabetic foot healing: a systematic review and meta-analysis. Int J Low Extrem Wounds. 2018;1534734618809583. doi:10.1177/1534734618809583

7. Ding H, Fu XL, Miao WW, Mao XC, Zhan MQ, Chen HL. Efficacy of autologous platelet-rich gel for diabetic foot wound healing: a meta-analysis of 15 randomized controlled trials. Adv Wound Care. 2019;8(5):195-207. doi:10.1089/wound.2018.0861

8. Winkley K, Stahl D, Chalder T, Edmonds ME, Ismail K. Risk factors associated with adverse outcomes in a population-based prospective cohort study of people with their first diabetic foot ulcer. J Diabetes Complications. 2007;21(6):341-349. doi:10.1016/j.jdiacomp.2007.09.004

9. Morbach S, Furchert H, Gröblinghoff U, et al. Long-term prognosis of diabetic foot patients and their limbs: amputation and death over the course of a decade. Diabetes Care. 2012;35(10):2021-2027. doi:10.2337/dc12-0200 
10. Xiong H, Yan P, Huang Q, et al. A prognostic role for non-thyroidal illness syndrome in chronic renal failure: a systematic review and meta-analysis. Int J Surg. 2019;70:44-52. doi:10.1016/j.ijsu.2019.08.019

11. Su W, Zhao XQ, Wang M, Chen H, Li HW. Low T3 syndrome improves risk prediction of in-hospital cardiovascular death in patients with acute myocardial infarction. J Cardiol. 2018;72(3):215-219. doi:10.1016/j.jjcc.2018.02.013

12. Todd SR, Sim V, Moore LJ, Turner KL, Sucher JF, Moore FA. The identification of thyroid dysfunction in surgical sepsis. $J$ Trauma Acute Care Surg. 2012;73(6):1457-1460. doi:10.1097/TA.0b013e318270db2c

13. Bassols J, Prats-Puig A, Soriano-Rodríguez P, et al. Lower free thyroxin associates with a less favorable metabolic phenotype in healthy pregnant women. J Clin Endocrinol Metab. 2011;96(12):3717-3723. doi:10.1210/jc.2011-1784

14. Yuan D, Zhang C, Jia S, et al. Predictive value of free triiodothyronine (FT3) to free thyroxine (FT4) ratio in long-term outcomes of euthyroid patients with three-vessel coronary artery disease. Nutr Metabol Cardiovasc Dis. 2021;31(2):579-586. doi:10.1016/j.numecd.2020.10.011

15. Yuan D, Jia S, Zhu P, et al. Usefulness of FT3 to FT4 ratio to predict mortality in euthyroid patients with prior cardiovascular events undergoing PCI: five-year findings from a large single-center cohort study. Front Endocrinol (Lausanne). 2021;12:700349. doi:10.3389/ fendo.2021.700349

16. Kozdag G, Ural D, Vural A, et al. Relation between free triiodothyronine/free thyroxine ratio, echocardiographic parameters and mortality in dilated cardiomyopathy. Eur J Heart Fail. 2005;7(1):113-118. doi:10.1016/j.ejheart.2004.04.016

17. Pasqualetti G, Schirripa M, Dochy E, et al. Thyroid hormones ratio is a major prognostic marker in advanced metastatic colorectal cancer: results from the phase III randomised CORRECT trial. Eur J Cancer. 2020;133:66-73. doi:10.1016/j.ejca.2020.04.023

18. Pasqualetti G, Calsolaro V, Bernardini S, et al. Degree of peripheral thyroxin deiodination, frailty, and long-term survival in hospitalized older patients. J Clin Endocrinol Metab. 2018;103(5):1867-1876. doi:10.1210/jc.2017-02149

19. Zou J, Li Z, Tian F, et al. Association between normal thyroid hormones and diabetic retinopathy in patients with type 2 diabetes. Biomed Res Int. 2020;2020:8161797. doi:10.1155/2020/8161797

20. Qin K, Zhang F, Wu Q, et al. Thyroid hormone changes in euthyroid patients with diabetes. Diabetes Metabol Syndr Obes. 2020;13:2533-2540. doi: $10.2147 /$ dmso.S260039

21. Zhang T, Shi J, Peng Y, et al. Sex-influenced association between free triiodothyronine levels and poor glycemic control in euthyroid patients with type 2 diabetes mellitus. J Diabetes Complications. 2020;34(11):107701. doi:10.1016/j.jdiacomp.2020.107701

22. Zou J, Tian F, Zhang Y, et al. Association between thyroid hormone levels and diabetic kidney disease in euthyroid patients with type 2 diabetes. Sci Rep. 2018;8(1):4728. doi:10.1038/s41598-018-22904-7

23. Wu J, Li X, Tao Y, Wang Y, Peng Y. Free triiodothyronine levels are associated with diabetic nephropathy in euthyroid patients with type 2 diabetes. Int J Endocrinol. 2015;2015:204893. doi:10.1155/2015/204893

24. Falkowski B, Rogowicz-Frontczak A, Grzelka A, et al. Higher free triiodothyronine concentration is associated with lower prevalence of microangiopathic complications and better metabolic control in adult euthyroid people with type 1 diabetes. Endocrine. 2018;60(3):458-465. doi:10.1007/s12020-018-1582-8

25. Levey AS, Stevens LA, Schmid CH, et al. A new equation to estimate glomerular filtration rate. Ann Intern Med. 2009;150(9):604-612. doi:10.7326/0003-4819-150-9-200905050-00006

26. Bravo-Molina A, Linares-Palomino JP, Vera-Arroyo B, Salmerón-Febres LM, Ros-Díe E. Inter-observer agreement of the Wagner, University of Texas and PEDIS classification systems for the diabetic foot syndrome. Foot Ankle Surg. 2018;24(1):60-64. doi:10.1016/j.fas.2016.10.009

27. Textor J, van der Zander B, Gilthorpe MS, Liskiewicz M, Ellison GT. Robust causal inference using directed acyclic graphs: the R package 'dagitty'. Int J Epidemiol. 2016;45(6):1887-1894. doi:10.1093/ije/dyw341

28. van Vliet NA, van der Spoel E, Beekman M, et al. Thyroid status and mortality in nonagenarians from long-lived families and the general population. Aging. 2017;9(10):2223-2234. doi:10.18632/aging.101310

29. Merke A, Merke J, Silbernagel G, März W. Free thyroid hormones and mortality in caucasians undergoing angiography: the Ludwigshafen Risk and Cardiovascular Health (LURIC) study. Endocr Pract. 2017;23(3):288-298. doi:10.4158/ep161217.Or

30. Maia AL, Goemann IM, Meyer EL, Wajner SM. Deiodinases: the balance of thyroid hormone: type 1 iodothyronine deiodinase in human physiology and disease. J Endocrinol. 2011;209(3):283-297. doi:10.1530/joe-10-0481

31. Peeters RP, Visser TJ. Metabolism of thyroid hormone. In: Feingold KR, Anawalt B, Boyce A, et al., editors. Endotext. South Dartmouth (MA): MDText.com, Inc; Copyright (C) 2000-2021, MDText.com, Inc. 2000.

32. Lee S, Farwell AP. Euthyroid sick syndrome. Compr Physiol. 2016;6(2):1071-1080. doi:10.1002/cphy.c150017

33. Roef GL, Rietzschel ER, Van Daele CM, et al. Triiodothyronine and free thyroxine levels are differentially associated with metabolic profile and adiposity-related cardiovascular risk markers in euthyroid middle-aged subjects. Thyroid. 2014;24(2):223-231. doi:10.1089/thy.2013.0314

34. McIver B, Gorman CA. Euthyroid sick syndrome: an overview. Thyroid. 1997;7(1):125-132. doi:10.1089/thy.1997.7.125

35. Park SY, Park SE, Jung SW, et al. Free triiodothyronine/free thyroxine ratio rather than thyrotropin is more associated with metabolic parameters in healthy euthyroid adult subjects. Clin Endocrinol (Oxf). 2017;87(1):87-96. doi:10.1111/cen.13345

36. Zoccali C, Mallamaci F. Thyroid function and clinical outcomes in kidney failure. Clin J Am Soc Nephrol. 2012;7(1):12-14. doi:10.2215/ cjn.12081111

37. Ogliari G, Smit RA, van der Spoel E, et al. Thyroid status and mortality risk in older adults with normal thyrotropin: sex differences in the Milan geriatrics 75+ cohort study. J Gerontol a Biol Sci Med Sci. 2017;72(4):554-559. doi:10.1093/gerona/glw113

38. Fortunato RS, Ferreira AC, Hecht F, Dupuy C, Carvalho DP. Sexual dimorphism and thyroid dysfunction: a matter of oxidative stress? $J$ Endocrinol. 2014;221(2):R31-40. doi:10.1530/joe-13-0588

39. Zhang LX, Wang YT, Zhao J, Li Y, Chen HL. Sex differences in osteomyelitis of the foot in persons with diabetes mellitus: a meta-analysis. Wound Manag Prev. 2021;67(5):19-25. doi:10.25270/wmp.2021.5.1925

40. Gussekloo J, van Exel E, de Craen AJ, Meinders AE, Frölich M, Westendorp RG. Thyroid status, disability and cognitive function, and survival in old age. JAMA. 2004;292(21):2591-2599. doi:10.1001/jama.292.21.2591

41. Jasim S, Gharib H. Thyroid and aging. Endocr Pract. 2018;24(4):369-374. doi:10.4158/ep171796.Ra

42. Morganti S, Ceda GP, Saccani M, et al. Thyroid disease in the elderly: sex-related differences in clinical expression. J Endocrinol Invest. 2005;28 (11Suppl Proceedings):101-104. 


\section{Publish your work in this journal}

Diabetes, Metabolic Syndrome and Obesity: Targets and Therapy is an international, peer-reviewed open-access journal committed to the rapid publication of the latest laboratory and clinical findings in the fields of diabetes, metabolic syndrome and obesity research. Original research, review, case reports, hypothesis formation, expert opinion and commentaries are all considered for publication. The manuscript management system is completely online and includes a very quick and fair peer-review system, which is all easy to use. Visit http://www.dovepress. com/testimonials.php to read real quotes from published authors.

Submit your manuscript here: https://www.dovepress.com/diabetes-metabolic-syndrome-and-obesity-targets-and-therapy-journal 